\title{
Anormalidades urinarias en pacientes adultos infectados por VIH.
}

Urinary abnormalities in adult patients infected with HIV.

\author{
Estremadoyro S Luis*, Montiel G Marco**, Lopez C Marga*, Loza M César*, \\ Hanawa K André*, Huaman H Carlos*, Gotuzzo H Eduardo**, Miyahira A Juan*.
}

*Servicio de Nefrología. Universidad Peruana Cayetano Heredia. Lima Perú.

**Instituto de Medicina Tropical Alexander Von Humboldt. Universidad Peruana Cayetano Heredia. Lima, Perú.

\section{RESUMEN}

Objetivo: Determinar la frecuencia de anormalidades urinarias en pacientes adultos infectados con el virus de la inmunodeficiencia humana (VIH) y los factores de riesgo asociados. Materiales y métodos: Se examinaron muestras de la primera orina matutina de 61 pacientes seropositivos (Western-Blot) atendidos en el consultorio externo del Instituto de Medicina Tropical Alexander von Humboldt entre noviembre 1993 y diciembre de 1994. Se determinó: osmolidad, $\mathrm{pH}$, examen bioquímico por tira reactiva, proteinuria en 24 horas (para pacientes con albuminuria $\geq 1+$ ) y sedimento urinario. Resultados: La edad promedio fue de 32,4 \pm 8,1 años, 58\% eran homosexuales, no hubieron adictos a drogas endovenosas y sólo un paciente era de raza negra. El 84\% tenía SIDA al momento del estudio. Se encontró por lo menos una alteración en el 27,9\% de los pacientes. El porcentaje de pacientes con incapacidad de concentración urinaria fue de 16,0\% y de acidosis tubular renal 13,3\%. Ningún paciente presentó proteinuria significativa, 3,3\% presentó hematuria 
glomerular y 4,9\% hematuria no glomerular. En ningún paciente se encontró glucosuria y se detectó leucocituria sólo en 3.3\% de ellos. No se encontró ningúna relación significativa entre variables clínicas y las alteraciones urinarias. Conclusión: La frecuencia de alteraciones urinarias asociadas a daño túbulo inetersticial fue de 29,7\%. La ausencia de proteinuria significativa obedece probablemente al escaso porcentaje de pacientes de raza negra y de adictos endovenosos. (Rev Med Hered 1996; 7: 108-112).

PALABRAS CLAVE: VIH, SIDA, anormalidades urinarias, proteinurias, hematuria, acidosis tubular renal.

\section{SUMMARY}

Objective: To determine the frequency of urinary abnormalities associated with human immunodeficiency virus (HIV) and their risk factors. Material and methods: We designed a descriptive, analytic and transversal study. We included 61 HIV outpatients (Western Blot positive) of the Instituto de Medicina Tropical Alexander von Humboldt and we determined osmolality, $\mathrm{pH}$, dipstick test and microscopic examination of urine sediment in fist morning urine collected with the midstream technique. Twenty four hours proteinuria was determined in patients with positive dipstick. Results: Age was $32.4 \pm 8.1$ years, 58\% were homosexuals. The sample was composed by non-addicts to intrevenoues drugs and only one patient was black, $84 \%$ of patients were AIDS. We found $27.9 \%$ patients with at least one urinary abnormality. Inability to urine concentration was found in $16 \%$, renal tubular acidosis in urinary acidosis in $13.3 \%$, leucocituria in 3.3\%, glomerular hematuria in $3.3 \%$, glomerular hematuria in 3.3\% and non-glomerular hematuria in $4.9 \%$. Protenuira and glucosuria were negative in all patients. No significative relation among clinical variables and urinary anormalities was demonstrated. Conclusions: Tubulo interstitial damageassociated urinary abnormalities were demonstrated in 29.7\%. Absence of proteinuria probably is due up to absence of intravenous drugs addicts and blacks. (Rev Med Hered 1996; 7: 108-112). 
KEY WORDS: HIV, AIDS, urinary abnormalities,urine, proteinuria, hematuria, renal tubular acidosis.

\section{INTRODUCCION}

La infección por el virus de la inmudeficiencia humana (VIH) es un problema de salud pública en el país. Según datos del Programa Especial de Control del SIDA del Ministerio de Salud, se hizo el diagnóstico de la enfermedad en 3289 pacientes hasta octubre de 1995. Esta cifra equivale al 22\% de todos los casos diagnosticados en los países del área andina y representa probablemente menos del 10\% del número real de personas infectadas (1).

Una de las complicaciones de la infección por VIH es la llamada nefropatía asociada al VIH (NAVIH), descrita en una frecuencia de $10 \%$ a $32 \%$ de todos los pacientes adultos seropositivos $(5,6)$. Puede presentarse en cualquier fase evolutiva de la infección VIH y su cuadro clínico típico es de síndrome nefrótico que progresa rápidamente a la insuficiencia renal crónica (5,7,8-12). Se han descrito otras formas de compromiso renal en los pacientes seropositivos, asociadas tanto a la infección viral como a infecciones oportunistas y uso de fármacos nefrotóxicos; entre estas se describen diversas anormalidades urinarias, producto de compromiso renal glomerular o túbulo intersticial $(8,10,13)$. Hasta el momento no se conocen datos epidemiológicos nacionales del compromiso renal en pacientes infectados por VIH.

El objetivo del presente estudio fue determinar la frecuencia de alteraciones urinarias en pacientes adultos infectados por VIH en nuestro medio e investigar los factores de riesgo asociados a ellas. 


\section{MATERIAL Y METODOS}

Se diseñó un estudio descriptivo y analítico de corte transversal. La población estudiada comprendió a pacientes seropositivos para VIH (confirmado por Western-Blot), que acudieron a la consulta externa del Instituto de Medicina Tropical Alexander Von Humboldt (IMTAH) de la Universidad Peruana Cayetano Heredia (UPCH), desde el $1^{\circ}$ de noviembre de 1993 hasta el 31 de diciembre de 1994 y aceptaron ser incluídos en el estudio. La muestra estudiada representó aproximadamente el 50\% de todos los pacientes seropositivos que consultaron durante el período de estudio.

Se obtuvieron muestras de la primera orina de la mañana de todos los pacientes incluidos en el estudio. Las muestras fueron procesadas antes de los primeros 15 minutos de obtenida la orina y se determinó osmolalidad, $\mathrm{pH}$, análisis bioquímico por tira reactiva y examen microscópico del sedimento urinario. Todas las muestras fueron procesadas en el laboratorio de investigación del servicio de Nefrología de la UPCH.

La osmolalidad se determinó por la técnica del punto de congelación, utilizando el osmómetro The Advanced Osmometer. Se repitió el examen en las mismas condiciones a todos los pacientes que tuvieron una osmolalidad urinaria menor de $500 \mathrm{mOsm} / \mathrm{Kg}$ de agua.

El pH urinario se determinó con un electrodo. Se repitió la determinación en una muestra de otro día si el pH urinario era mayor a 5,5. Se realizó test de acidificación con carga oral de cloruro de amonio (0,1 g/Kg de peso) y análisis de gases arteriales en los pacientes en quienes se obtuvo dos determinaciones de $\mathrm{pH}$ urinario mayor a 5,5.

A los pacientes que presentaron proteinuria igual o mayor a $1+$ por tira reactiva, se les determinó la proteinuria en orina de 24 horas por el método de precipitación con ácido fosfotúngstico (método de Tsuchiya).

El estudio microscópico de orina fue estudiada convencionalmente a 100 y 400X. En caso de encontrarse hematuria, se determinó el porcentaje de hematíes desmórficos con el 
microscopio de contraste de fases del IMTAH. A todos los pacientes con leucocituria se les realizó cultivo de orina para gérmenes comunes en el Laboratorio de Microbiología del IMTAH.

Utilizamos las siguientes definiciones para las alteraciones urinarias:

1. Proteinuria significativa $\geq 150 \mathrm{mg} / 24$ horas.

2. Hematuria: $>2$ hematíes/campo de $400 X$.

3. Hematuria glomerular : cilindros hemáticos o mas de 30\% de hematíes dismórficos en 2 muestras distintas.

4. Leucocituria: $>5$ leucocitos/campo de 400X.

5. Glucosuria renal: $\geq 1+$ en presencia de glicemia $<180 \mathrm{mg} / \mathrm{dl}$.

6. Incapacidad de concentración urinaria: Osmolalidad urinaria $<500$ en dos muestras tomadas después de 8 horas de restricción hídrica.

7. Incapacidad de acidificación urinaria: $\mathrm{pH}>5.5$ en dos muestras de la primera orina de la mañana.

8. Acidosis tubular renal: $\mathrm{pH}$ urinario $>5.5$ en presencia de bicarbonato sérico $<20$ $\mathrm{mEq} / \mathrm{L}$ (espontáneo o inducido por la prueba de acidificación).

Las características clínicas de la infección por VIH se definieron de la siguientes manera:

1. Forma de adquisición del VIH: sexual, sanguínea, no documentada.

2. Factores de riesgo: Homosexual, promiscuidad sexual, ser pareja de seropositivo, transfusiones, uso de drogas EV, desconocido.

3. Estadío de enferdedad: clasificación CDC (14).

Para el análisis estadístico se consideraron las siguientse variables:

1. Variables dependientes: resultados de los exámentes de orina. 
2. Variables independientes: Edad, sexo, raza, forma de adquisición de VIH, factores de riesgo, estadío de la enfermedad, tiempo desde el diangnóstico de seropositividad, infecciones asociadas a drogas que recibía al momento de la evaluación.

El análisis estadístico se hizo utilizando el paquete EPI-INFO v 5.0. Las relaciones entre variables continuas y discretas fueron estudiadas por la prueba de Mann-Whitney y las diferencias entre proporciones por la prueba exacta de Fischer. Se consideró estadísticamente significativo un valor de $\mathrm{p}<0,05$.

\section{RESULTADOS}

Se estudiaron 61 pacientes (54 varones y 7 mujeres), con edad promedio de 32,4 \pm 8,1 años. Un paciente era de raza negra, uno caucásico y el resto mestizos.

La forma de adquisición de la enfermedad fue por vía sexual en $85.2 \%$ de los casos y desconocída en el resto. El 58\% de los pacientes tuvo como factor de riesgo para contraer la infección a la homosexualidad, 27\% refirió promiscuidad sexual, 13\% fue pareja de persona seropositiva y $12 \%$ no tuvo ningún factor conocido de riesgo. No hubo adictos a drogas endovenosas.

El tiempo promedio transcurrido desde el diagnóstico serológico fue de 15.4 meses (1 a 94 meses). El estadío de enfermedad según la clasificación de la CDC, era II en 8\%, III en 8\% y IV (estadío SIDA) en 84\%.

Al momento del estudio tenían diagnóstico de tuberculosis 11 pacientes, 8 neumonía por $P$. Carinii, 5 isosporiasis, 7 criptococosis, 2 infección por citomegalovirus y 1 toxoplasmosis.

Los 11 pacientes con TBC estaban en tratamiento anti-tuberculoso. Otros tratamientos farmacológicos incluyeron: 10 pacientes que recibían sultas, 7 anfotericinas $B, 5$ zidovudina (AZT), 4 didanosina (DDI), 1 fluconazol y 1 cefalosporina. 
La prevalencia de alteraciones urinarias en la población estudiada se muestra en la figura $\mathrm{N}^{\circ} 1$. El urocultivo para gérmenes comunes fue positivo (E.coli $>10^{5} \mathrm{UFC} / \mathrm{ml}$ ) en uno y negativo en el otro paciente con leucocituria. El único paciente en eque se encontraron anormalidades urinarias sin estar en estadío IV fue el diagnosticado como portador de infección urinaria.

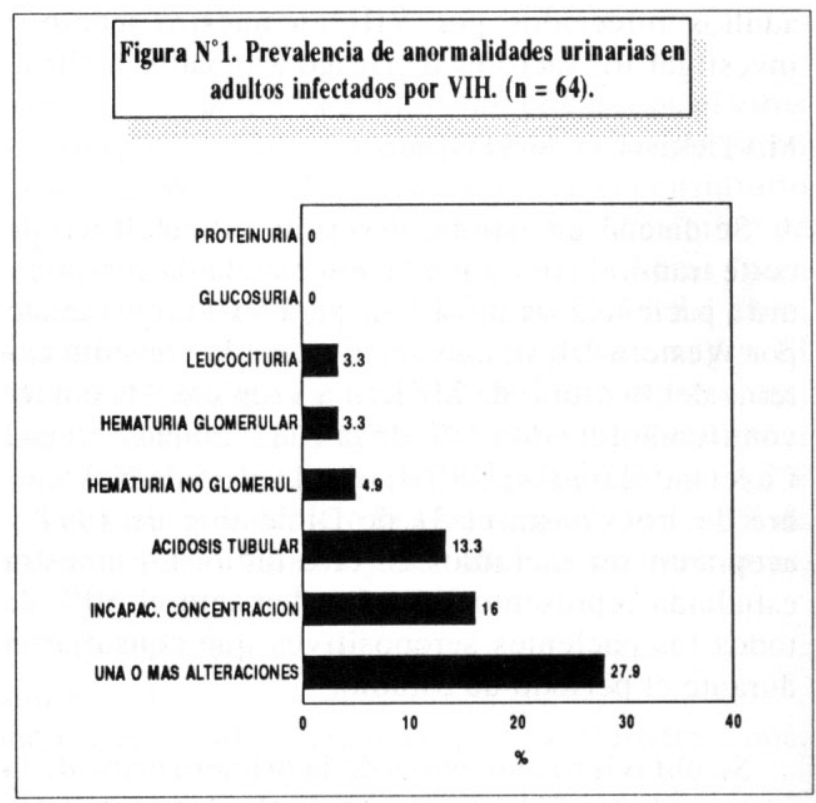

No se encontró ninguna asociación significativa entre las variables clínicas estudiadas (edad, sexo, raza, forma de adquisición del VIH, estadío CDC, infecciones asociadas, tiempo desde el diagnóstico de seropositividad, medicación recibida) y las alteraciones urinarias.

\section{DISCUSION}

Tres años después de publicados los primeros reportes de la infección humana por VIH en 1981, se describió la presencia de compromiso renal en un número significativo de pacientes afectados (2). El cuadro clásico se caracteriza por ser una glomeruloesclerosis 
focal y segmentaria que se manifiesta clínicamente como síndrome nefrótico, normotensión arterial, deterioro rápido de la función renal y riñones de tamaño normal o incluso grandes. Ha sido descrita no sólo en el estadío IV de enfermedad (SIDA), presentándose más de 50\% de los casos en los estadíos pre-SIDA: seropositivo asintomático (II) y complejo relacionado al SIDA (III). Con menor frecuencia se han descrito otras glomerulopatías como enfermedad a cambios mínimos, glomerulonefritis membrano-proliferativa y nefropatía por IgA, asociadas a la infección por VIH (5,7,8,9,10,11,12).

Los factores de riesgo reconocidos asociados a nefropatía son la raza negra (riesgo relativo 10:1), el sexo masculino y la adicción a heroína (50\% de los pacientes afectados son adictos) $(8,10,11,12)$. El compromiso glomerular heroinómano era ya conocido antes de 1981. Se manifiesta como síndrome nefrótico, pero no suele comprometer la función renal en forma tan rápida como la NAVIH, la mayoría de pacientes son hipertensos y los riñones tienden a ser pequeños. Histológicamente es una glomeruloesclerosis focal y segmentaria que se diferencia de la NAVIH por la menor frecuencia de alteraciones tubulares ultraestructurales conocidas como inclusiones túbulo-reticulares, que probablemente representen partículas virales $(8,10,1,15)$.

En la patogénesis de la NAVIH parece jugar un rol central la infección de células renales por el VIH. Con técnicas de hibridación del DNA, se ha demostrado la presencia del genoma viral en células glomerulares y tubulares demás de 90\% de pacientes con NAVIH, siendo en cambio rara su detección en pacientes seropositivos sin compromiso renal. Sin embargo, no se descarta la posibilidad de que la sobreinfección por otros virus o microorganismos como el Mycoplasma fermentans (cepa incognitus), el abuso de drogas, y otros factores genéticos e inmunológicos, tengan también importancia en la génesis del daño renal $(10,16,17)$.

El IMTAH de la UPCH es un centro nacional de referencia para pacientes infectados por VIH, cubriendo aproximadamente 25\% de la demanda de atención de la ciudad de Lima. 
Las características demográficas de la muestra estudiada son similares a las descritas para el universo nacional. El 80.5\% de pacientes notificados al Programa Nacional de Control de SIDA y enfermedades de transmisión sexual (PROCETS) del Ministerio de Salud, tiene de 20 a 49 años y el 87.2\% son de sexo masculino (1).

Ninguno de los pacientes evaluados en el estudio tubo síndrome nefrótico. Al evaluar un marcador más sensible de daño renal, como la proteinuria signigicativa, tampoco encontramos ningún paciente afectado. El uso de la tira reactiva podría haber provocado falsos negativos. La sensibilidad del resultado mayor de 1+ por tira reactiva para detectar proteinuria real ha sido descrita entre $60-80 \%(18,19)$. Sin embargo, la sensibilidad de nuestro estudio es mayor a esa cifras, ya que se realizó dosaje de proteinuria en 24 horas a todos los pacientes con resultados $1+$.

Aún con las limitaciones descritas, nuestra realidad es muy distinta a la de poblaciones con altos porcentajes de pacientes de raza negra y adictos endovenosos. Un estudio de 75 pacientes seropositivos de la consulta externa de un hospital de Miami, mostró que 43\% tenía proteinuria mayor de $500 \mathrm{mg} / 24$ horas y en 9\% la proteinuria alcanzaba el rango nefrótico (10). En otra serie de 86 pacientes infectados y seguidos en la consulta externa de un hospital de Brooklyn, se encontró proteinuria significativa en 58\% de ellos (19). Soni y colaboradores encontraron proteinuria en $46.7 \%$ de 182 pacientes del Bronx en Nueva York, teniendo la mayoría de ellos más de 2 gramos en 24 horas (6). En cambio, series de San Francisco, Washington, Dallas o Amsterdam, con bajo porcentaje de pacientes de raza negra y adictos endovenosos, muestran prevalencias de proteinuria menores al 3\% $(8,10,11,21,22)$.

El hallazgo de hematuria con hematíes dismórficos tiene una sensibilidad y especificidad mayor del 90\% para el diagnóstico de hematuria glomerular $(23,24)$. La confirmación de glomerulopatía en estos casos requiere de estudio histopatológicos, pero ninguno de los dos pacientes a quienes se detectó hematuria aceptó ser sometido a biopsia renal. 
El daño glomerular no es la única forma de compromiso renal en el paciente infectado por VIH. Si bien no se ha descrito una entidad específica caracterizada por daño renal predominantemente túbulo intersticial asociada a la infección por VIH, se han reportado porcentajes significativos de pacientes con este tipo de anormalidades. En la autopsias de 50 pacientes VIH positivos de hospitales universitarios de Texas se encontró que en más del 65\% de las muestras habían cilindros tubulares, nefrocalcinosis en casi el 40\% además porcentajes significativos de compromiso por citomegalovirus, criptococosis, histoplasmosis e infección por micobacterias atípicas (13). El daño intersticial se explica probablemente no sólo por infecciones oportunistas, sino también por la exposisción a drogas nefrotóxicas. Es bien conocida la asociación entre sultas e isoniazida, rifampicina, anfotericina B y betalactámicos con nefritis tubulointersticial (25).

En la muestra estudiada encontramos un porcentaje elevado de anormalidades tubulointersticiales, evidenciadas por defectos en la concentración y acidificación urinaria, así como hematuria noglomerular y leucocituria. A exceptción del paciente con infección urinaria por gérmenes comunes, ningún paciente en estadío pre-SIDA (libre de infecciones oportunistas y de drogas nefrotóxicas) presentó alteraciones urinarias. Esto abonaría a favor de la importancia de estos factores de riesgo para el desarrollo de nefropatía intersticial. El no haber encontrado asociación estadísticamente signigicativa entre el pertenecer al estadío pre-SIDA y no presentar anormalidades tubulares, podría deberse al tamaño de nuestra muestra.

En conclusión, al tratar pacientes adultos infectados con VIH, debe tenerse presente que un porcentaje con VIH, debe tenerse presente que un porcentaje cercano al 30\% presentan alguna anormalidad urinaria, predominantemente de tipo túbulo intersticial. En cambio, de manera similar a lo descrito en otras series con bajo porcentaje de pacientes de raza negra y adictos endovenosos, encontramos una frecuencia muy baja de anormaliades urinarias secundarias a compromiso glomerular. 


\section{Agradecimientos:}

Este estudio fue financiado por el Laboratorio de Investigación de Nefrología de la Universidad Peruana Cayetano Heredia.

Al Laboratorio de Microbiología del Instituto de Medicina Tropical Alexander Von Humboldt, por su apoyo en los cultivos de orina.

\section{REFERENCIAS BIBLIOGRAFICAS}

1.Programa Nacional de Control de SIDA y enfermedades de transmisión sexual. Informe de Octubre de 1995. Lima, Peru:Ministerio de Salud del Perú; 1995.

2.Sánchez J, Mazzotti G, Cuéllar L, Campos P, Gotuzzo E. SIDA: Epidemiología, diagnóstico, tratamiento y control de la infección VIH/SIDA. Lima, Perú: Organización Mundial de la Salud Vía Libre; 1995.

3.Sánchez J, Vega O, Cuellar L, et al. Epidemiological trends of HIV infection in Peru. Resumen PO-CO6-2722. Amsterdam:XI International Conference on AIDS; 1991.

4.Sanchez J, Gotuzzo E, Escamilla J, et al. STD in female sex workers: Reduced by regular condon use but not by participation in STD control program. Book of proceedings tenth international meeting. Helsinki, Finlandia: Internacional Society for STD Research; 1993. 5.Rao TKS, Filippone EJ, Nicastri AD, et al. Associated focal and segmental glomeruloesclerosis in the acquired immunodeficiency Syndrome. N Eng J Med 1984; 310: 669-673.

6.Soni A, Agarwal A, Chander P, et al. Evidence for an HIV-related nephropaty: a clinicopathological study. Clin Nephrol 1989; 31: 12-17.

7.Carbone L, D’Agati V, Cheng JT, Appel G. Course and prognosis of Human Immunodeficiency Virus-Associated Nephropaty. Am J Med 1989; 87: 389-395.

8.Glassock RJ. HIV infection and the Kidney. Ann Int Med 1990; 112: 35-49.

9.Langs C, Gallo GT, Schacht RG, Sidhu G, Baldwin DS. Rapid Renal Failure in AIDSAssociated Focal Glomeruloesclerosis. Arch Int Med 1990; 150: 287-292. 
10.Gardenswartz MH, Rao TKS. Renal diseases associated with Human Immunodeficiency Virus (HIV) Infection (HIV- Associated Nephropaty). En: Schrier RW and Gottschalk CW (ed). Diseases of the Kidney. Boston: Little, Brown and Co.; 1993.

11.Kimmel PL, Berns JS. Renal and Urologic aspects of HIV infection. Contemporary Issues in Nephrology. New York: Churchill Livingstone; 1995.

12.Humphreys MH. Human immudeficiency virus-associated glomeruloesclerosis. Kidney Int 1995; 48: 311-320.

13.Seney FD, Burns DK, Silva FG. Acquired Immunodeficiency Syndrome and the Kidney. Am J Kidney Dis 1990; 16: 1-13.

14.Center for Disease Control. CDC classification of spectrum of disease produced by HI.V infection. MMWR 1987; 36: 235-236.

15.Rao TKS, Nicastri AD, Friedman EA. Natural history of heroin associated nephropaty. N Eng J Med 1974; 290: 19-22.

16.Kimmel PL, Ferreira-Centeno A, Farkas-Szallasi T, Abram AA, Garret CT. Viral DNA in microssected renal biopsy tissue from HIV infected patients with nephrotic syndrome. Kidney Int 1993; 43: 1347-1352.

17.Kimmel PL, Philips M, Ferreira-Centeno A, Farkas-Szallasi T, Abraham AA, Garret CT. HIV-associated immunemediated renal disease. Kidney Int 1993; 44: 1327-1340.

18.Fairley KF. Urianalysis. En: Schrier R, Gottschalk C. (ed). Diseases of the kidney. Boston: Little, Brown and Co; 1993.

19.Meyer LM, Mercer BM, Friedman SA, Sibai BM. Urinary dipstick protein: A poor predictor of absent or severe proteinuria. Am J Obstet Gynecol 1994; 170: 137-141.

20.De Alvaro F, Lundin P, Chirgwin K, et al. Proteinuria in HIV infected patients. J Am Soc Nephrol 1990; 1: 306-309.

21.Mazbar S, Humphreys MH. AIDS-associated nephropaty is not seen at San Francisco General Hospital. Kidney Int 1988; 33: 202.

22.Humphreys MH. Human Immunodeficiency Virus Associated Nephropaty. East is East and West in West? Arch Int Med 1990; 150: 253-255.

23.Fairley KF, Birch DF. Hematuria: A simple method for identifying glomerular bleending. Kidney Int 1982; 21: 105-108. 
24.Fassett RG, Horgan BA, Mathew TH. Detection of glomerural bleending by phasecontrast microscopy. Lancet 1982; I: 1432-1434.

25.Bennett WM, Elzinga LW, Poter GA. Tubulointersticial Disease and Toxic Nephropaty. En: Brenner BM, Rector FC (eds). The Kidney. Philadelphia: WB Saunders Co; 1991. p. 1430-1496.

\section{Correspondencia:}

Dr. Luis Estremadoyro S.

Servicio de Nefrología, Universidad Peruana Cayetano Heredia.

Av. Honorio Delgado 430, San Martín de Porres.

Lima, Perú. 